\title{
Un vent nouveau pour les éditions Robert
}

\author{
Jean-Nicolas De Surmont, University of Leeds, Leeds, United Kingdom \\ (jdesurmont@yahoo.fr)
}

Après la réédition de ses produits électroniques (Petit Robert électronique 2007 paru en septembre 2006 et le Grand Robert électronique paru en 2005) mentionnons la récente parution de deux ouvrages: Le Dictionnaire des combinaisons de mots: les synonymes en contexte (collection les Usuels, 2007, 1011 pages) et le Robert Van Dale Dictionnaire français-néerlandais; néerlandais-français. Il convient néanmoins de signaler que l'une des particularités des récentes éditions Robert consistera en l'ajout de 48 belgicismes s'appuyant en partie sur un travail que nous avons effectué au Centre de recherche Valibel en 2003 et 2004." Cette nouveauté belge dans le Petit Robert fait suite à notre suggestion aux éditions Robert en 2003 de revoir la nomenclature des belgicismes dans les dictionnaires Robert. En outre, à la demande de Madame Durand, récemment nommée à la direction des éditions Robert, et qui voulait se mettre au goût du jour en vue du festival de la Francophonie de 2006 en France, le Centre de recherche Valibel a été prié de livrer le fruit de ses recherches entamées depuis 2003 et qui a fait l'objet de rencontres nombreuses. Le Festival de la Francophonie constituait en quelque sorte une réaction contre des commentaires selon lesquels la France s'excluerait du mouvement de la Francophonie. (Voir notre article paru sur http://www.lianes.org). Le Petit Larousse illustré a tenté de renverser la tendance en incluant la France dans les activités de la Francophonie en 2006.

Cette volonté de prendre en compte les topolectismes est également présente dans le Dictionnaire bilingue français-néerlandais/néerlandais-français dirigé par Paul Bogaards et qui en est à sa quatrième édition. La publication d'un dictionnaire bilingue de ce type nous paraît primordiale dans un pays comme la Belgique, dont les deux langues les plus importantes administrativement parlées sont le français et le flamand, ainsi qu'en Hollande où la pénétration $\mathrm{du}$ français remonte, $\mathrm{du}$ moins dans les classes dominantes à plusieurs siècles, et où sont publiés au XVIIe siècle, les dictionnaires de Furetière et Bayle. Parmi les 700 mots nouveaux de ce dictionnaire, dont la première édition remonte à 1988, mentionnons l'ajout de flandricismes, qu'on appelle parfois à tort belgicisme alors que dans la terminologie métalinguistique il s'agit en fait de particularités francophones. On note dans le flamand de Belgique quelques flandricismes comme les toponymes (Rijsel pour Lille par exemple). C'est surtout le néerlandais qui a été mis à jour compte tenu de la nouvelle orthographe, quant à la partie française, notamment pour les belgicismes, on a considéré que le fonds du Petit Robert était suffisant. 
Contrairement au dictionnaire Robert Collins, le dictionnaire bilingue français/néerlandais est monodirectionnel et s'adresse essentiellement aux francophones de Belgique. Le Dictionnaire Van Dale/Robert, très connu, s'est vendu à 10000 exemplaires pour sa première édition, essentiellement en Belgique, alors que le Petit Robert se vend annuellement à environ 200000 exemplaires en France. Parmi les ajouts en langue française mentionnons courriel, arobase, chat, texto, teleachat, e-commerce, placoplâtre et du côté flamand: drukkingsgroep, schoollopen, inpikken op, onthaal, bissen, traduit par la métalangue belge ce qui est exceptionnel. L'ajout de courriel nous semble intéressant puisqu'il témoigne de la reconnaissance d'une recommandation québécoise à l'échelle internationale au détriment de Mél qui est en perte d'usage.

Récemment présenté au Salon du livre de Paris de 2007, Le Dictionnaire de combinaisons de mots des éditions Robert est paru le 15 mars 2007. Il est le premier dictionnaire de ce type depuis 250 ans selon l'affirmation de Dominique Le Fur qui a dirigé l'ouvrage. Il comporte 160000 combinaisons de mots classées par sens accompagnées de 26000 exemples. Il s'agit en fait de syntagmes à figement assez important dans la langue comme santé de fer, faire entorse à une loi, candidat de poids, traduire en justice, harcèlement policier, discrimination institutionnelle, etc. Le dictionnaire a nécessité trois ans de travail et s'appuie sur un corpus de 500000 millions de mots-occurrences.

Parmi les prochaines productions des éditions Robert figurera l'édition de poche du bilingue néerlandais/français et la remise à jour du Super Senior Collins/Robert.

Mentionnons que la Belgique a non seulement fait un accueil favorable aux récents dictionnaires Robert mais également à la publication du Dictionnaire des Belgicismes de Gorges Lebouc (Bruxelles, Éditions Racine, 2006, préface d'Henriette Walter), et qui s'appuie sur un corpus tiré du journal Le Soir et présentant parfois les emplois similaires de certains belgicismes en France et au Québec. Dans ce cas de nombreuses réserves sont à émettre. La présentation des belgicismes en contexte, dans un texte à thème bien ciblé, nous semble fort intéressant sur le plan pédagogique.

\section{Note}

$\dagger \quad$ Le site du Centre de recherche Valibel à Louvain-la-Neuve, dirigé par Michel Francard, indique que cela est paru dans l'édition 2007 du Petit Robert mais c'est en fait de l'édition 2008 qui paraîtra en août 2007 qu'il s'agit. En effet en cette année 2006 ne sont ajoutés à la nomenclature du Petit Robert que quelques belgicismes. 